OPEN ACCESS

Edited by:

Linxin Li,

University of Oxford, United Kingdom

Reviewed by:

Minshu Li,

Tianjin Medical University General

Hospital, China

Songjie Liao,

The First Affiliated Hospital of Sun

Yat-sen University, China

Muyassar Mamtilahun,

Shanghai Jiao Tong University, China

${ }^{*}$ Correspondence:

Anding Xu

tli@@jnu.edu.cn

Specialty section:

This article was submitted to

Stroke,

a section of the journal

Frontiers in Neurology

Received: 04 June 2021

Accepted: 26 August 2021

Published: 22 September 2021

Citation:

Tan Z, Zhao Y, Yang W, He S, Ding Y and Xu A (2021) Efficacy and Safety of

Adherence to dl-3-n-Butylphthalide

Treatment in Patients With

Non-disabling Minor Stroke and

TIA-Analysis From a Nationwide,

Multicenter Registry.

Front. Neurol. 12:720664.

doi: 10.3389/fneur.2021.720664

\section{Efficacy and Safety of Adherence to dl-3-n-Butylphthalide Treatment in Patients With Non-disabling Minor Stroke and TIA-Analysis From a Nationwide, Multicenter Registry}

\author{
Zefeng Tan ${ }^{1,2}$, Yin Zhao ${ }^{1}$, Wanyong Yang ${ }^{1}$, Shenwen $\mathrm{He}^{2}$, Yan Ding ${ }^{1}$ and Anding $\mathrm{Xu}^{1 *}$ \\ on behalf of the BRIDGE trial Investigators
}

\begin{abstract}
'Department of Neurology, the First Affiliated Hospital, Jinan University, Guangzhou, China, ${ }^{2}$ Department of Neurology, Shun De Hospital of Jinan University, Guangzhou, China
\end{abstract}

Background: DI-3-n-Butylphthalide (NBP) has the potential to improve clinical outcomes in acute ischemic stroke patients by improving collateral circulation. We aimed to evaluate the efficacy and safety of NBP in patients with non-disabling minor ischemic stroke and transient ischemic attack (TIA).

Methods: The BRIDGE (the observation study on clinical effectiveness of NBP on patients with non-disabling ischemic cerebrovascular disease) is a prospective registry to monitor the efficacy and safety of NBP therapy in acute non-disabling ischemic stroke or high-risk TIA. Non-disabling minor ischemic stroke patients within $48 \mathrm{~h}$ were enrolled across 51 stroke centers in China. We divided patients into NBP compliance or non-compliance groups according to their adherence to NBP. The primary outcome was the favorable functional outcome at 90 days, defined as a modified Rankin scale $(\mathrm{mRS})<2$.

Results: Between 10th October 2016 and 25th June 2019, 3,118 patients were included in this analysis. In multivariable analysis, after adjusting for common risk factors and demographic factors, NBP-compliance group has a higher proportion of favorable functional outcome (92.1 vs. $87.4 \%$, adjusted odds ratio 2.00 , 95\% confidence interval, $1.50-2.65)$, and a higher stroke recurrence rate (2.40 vs. $0.31 \%$, adjusted odds ratio 8.86, 95\% confidence interval, 3.37-23.30) than the NBP-non-compliance group. There was no significant difference in death and intracranial hemorrhage rate between the two groups. In subgroup analysis, patients with National Institutes of Health Stroke Scale (NIHSS) scores from 3 to 5 who complied to NBP therapy had a higher rate of favorable functional outcomes than the NBP-non-compliance group. [88.82 vs. $76.21 \%$, adjusted odds ratio $2.52(1.81-3.50)$, adjusted interaction $P=0.00]$. 
Conclusion: In non-disabling minor ischemic stroke or TIA patients, compliance with NBP therapy led to better 90-day functional outcomes despite a higher risk of recurrence, and this effect seems to be stronger in patients with NIHSS scores of 3-5. Further large randomized, double-blind controlled studies to analyse the association between NBP and functional outcome is warranted in the coming future.

Keywords: minor stroke, dl-3-n-butylphthalide, TIA, efficacy, modified rankin scale, non-disabling

\section{INTRODUCTION}

Studies have reported that minor stroke and high-risk TIA (ABCD2 score $\geq 4$ ) may have a high risk of early stroke recurrence and a more unsatisfactory clinical outcome than expected (1-4). Although treatment of minor stroke and highrisk TIA has improved, the risk of stroke recurrence within 90 days of stroke onset has been reported to be as high as 10$20 \%$ (1-4). Randomized, double-blind controlled studies based on Chinese populations have reported a 90 -day fatal or disability incidence of $6.03 \%$ and a 90 -day stroke recurrence rate of $9.6 \%$ in patients with minor stroke [National Institutes of Health Stroke Scale (NIHSS), 0-3] or high-risk TIA (5).

Evaluating collateral circulation at an early stage will facilitate the selection of intravenous thrombolysis and endovascular therapy (6-8). More importantly, a robust collateral flow will compensate for blood supply in the ischemic area, reduce core infarct volume and revive the penumbra zone.

Dl-3-n-butylphthalide (NBP) is a synthetic compound based on 1-3-n-butylphthalide extracted from the seeds of Apium graveolens Linn. Synthesized NBP has been approved to treat ischemic stroke patients with medium and small infarct foci in China (9). NBP improved collateral circulation by restoring the diameter of meningeal micro-arteries in the ischemic area and promoting blood vessel formation in mice $(10,11)$. Results of several multicenter randomized, double-blind, placebocontrolled trials evaluating oral NBP in patients with acute ischemic stroke showed significant improvements in neurological deficits and activity of daily living scales in the NBP -treated group compared with the placebo-controlled group, with a good safety profile $(9,12)$, and accepted by the Chinese guidelines for acute ischemic stroke (13). However, real-world, large-scale clinical studies of NBP therapy in patients with non-disabling minor stroke and high-risk TIA are still lacking.

This study aimed to analyze the efficacy and safety of NBP in patients with Non-disabling minor ischemic stroke and highrisk TIA.

\section{METHODS}

\section{The BRIDGE Registry}

The BRIDGE (the observation study on clinical effectiveness of NBP on patients with non-disabling ischemic cerebrovascular disease) is a prospective registry that was initiated in 2018 to monitor efficacy and safety of NBP therapy in acute nondisabling ischemic stroke (NIHSS $\leq 5$ on admission) or high-risk TIA ( $\mathrm{ABCD} 2$ score $\geq 4$ on admission). For the BRIDGE registry, specific information on timing and dosing of NBP, antiplatelet and statin therapy, and other stroke risk factors was added to the standard questionnaire. Data presented were obtained by standardized questionnaires in the 51 participating hospitals, medical history (prior coronary interventions, congestive heart failure, diabetes mellitus, renal insufficiency), biochemical test results, stroke subtype, and brain imaging. The clinical research forms available for the study were centralized at NCRC-ND (The China National Clinical Research Center for Neurological Diseases) and recorded into the Electronic Data Capture System [EDC 1.0, Jianhe Jiuzhou (Beijing) Technology Co.]. All data were analyzed centrally at NCRC-ND, China. Local monitoring and audits of the data have been performed in $10 \%$ of randomly selected patients. The ethics committee has approved the registry study. The local ethics committee has approved the registry of each center. Trial registration: http://www.chictr.org. cn/ Identifier: ChiCTR-OPC-16008069.

\section{Patient Selection}

All patients with non-disabling ischemic stroke (NIHSS $\leq$ 5 on admission) or high-risk TIA (ABCD2 score $\geq 4$ on admission) within $48 \mathrm{~h}$ of onset and treated with NBP were included on an intention-to-treat basis. Exclusion criteria include: (1) intracranial hemorrhage or vascular malformation, tumor, abscess, or other common non-ischemic brain diseases (e.g., multiple sclerosis); (2) iatrogenic stroke; (3) patients receiving early recanalization therapy (intravenous thrombolysis or endovascular thrombectomy); (4) pre-morbid mRS score $>2$; and (5) severe multi-organ failure; (6) patients with severe mental disorders and dementia; (7) patients who are pregnant or nursing or planning to become pregnant. All patients underwent cranial MRI during hospitalization to determine cerebral infarction diagnosis and exclude mimic stroke.

\section{Procedure and NBP Medication Compliance}

The $600 \mathrm{mg}$ dose of NBP was taken as two capsules three times daily, each containing $100 \mathrm{mg}$ of NBP. We invited all patients to take part in the study. Physicians contacted patients to obtain informed consent. All patients who agreed to participate in the study were instructed to take standard dose of NBP for 90 days and asked to complete a questionnaire at baseline; they were followed up every 30 for 90 days (Figure 1: flow progress diagram). For each month follow-up period, the patient received a pack of 180 capsules, and they were instructed to return the unused capsules at each visit. Nurses interviewed the patients. Patients were asked to record the duration of any lapses, the 


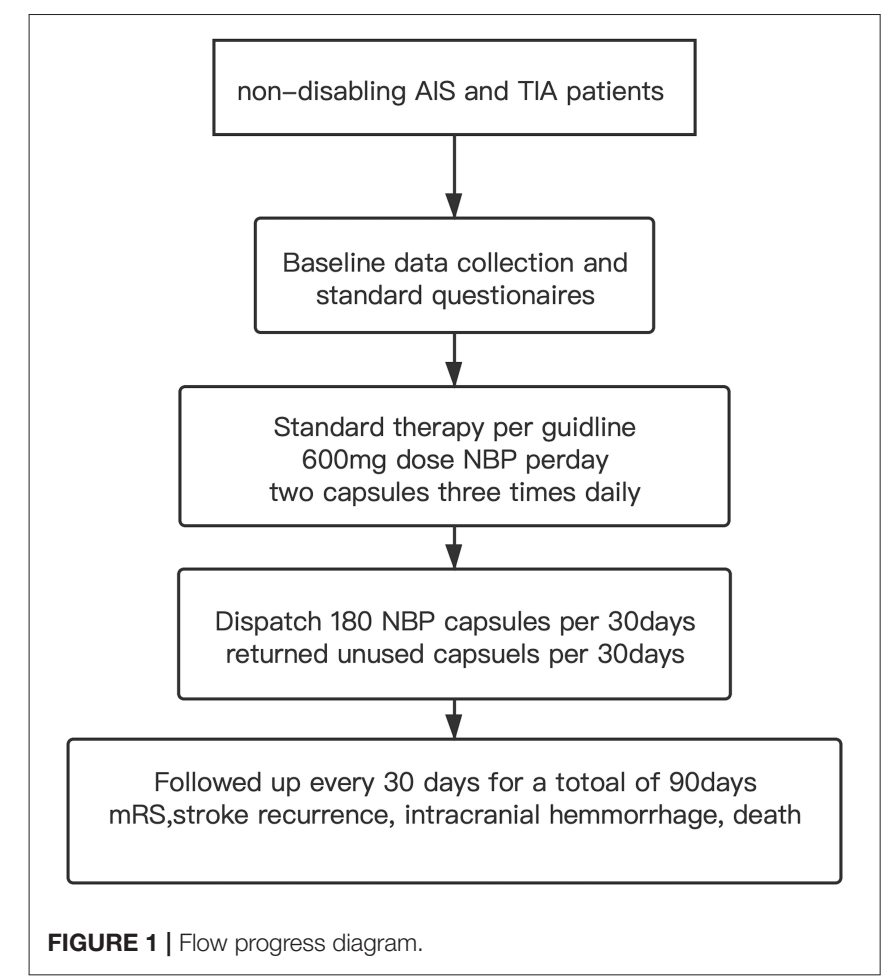

altered dosages that may have been taken, and the frequency of omitting any single dose. The answers were recorded in a completed CRF form for double-checking of the unreturned pills by follow-up personnels. Compliance with medication was calculated as the number of nonreturned capsules divided by six times the number of days in the follow-up period. The daily capsule count was expressed as a percentage of the scheduled dose (14). NBP medicine compliance was defined as taking $80 \%$ or more of the prescribed NBP doses (15). We monitored the patient's adverse reactions in accordance with the usual followup requirements, and the patient's liver and kidney function monitoring is carried out during baseline and routine follow-up.

\section{Sample Size}

Assuming the proportion of good functional outcome (mRS0-1) in NBP-compliance group is $85 \%$, and in NBP-non compliance group is $80 \%$, power of 0.90 , and a level of 0.05 , we required 1,209 participants per group. To account for possible dropouts of $10 \%$, we increased the sample to 2,688 .

\section{Statistical Methods}

All statistical analyses were performed using SAS version 9.4, and continuous variables are expressed as mean and standard deviation or median and quartiles.

We present data as absolute numbers or percentages. The Pearson-Fisher $\chi^{2}$ test compared the frequencies of categorical variables in the two treatment groups. The Mann-WhitneyWilcoxon test compared continuous variables.

We compared the proportion of stroke recurrence at 90 days between the two groups using Fisher's exact probability test or $\chi^{2}$ test. We calculated HRs and $95 \%$ confidence intervals using single-factor and multifactor Cox regression.

We compared the proportion of favorable outcomes ( $\mathrm{mRS} \leq$ 1) between the two groups using single-factor and multifactor regression analysis.

We used single and multivariable Cox regression to compare the proportion of death and intracranial hemorrhage between the two groups at 90 days and calculate $\mathrm{HR}$ and 95\% confidence intervals. A propensity score for NBP-compliance treatment was created to adjust for baseline differences between NBP-compliance and NBP-non-compliance subjects. A logistic regression model with compliance -NBP treatment as the outcome was used to generate propensity scores for all subjects. Variables that are potential confound compliance for NBP were included in three models; model 1 (age and sex); model 2 (age, sex, history of hypertension, body mass index, history of diabetes, history of dyslipidemia, smoking, alcohol consumption, admission NIHSS score, and TOAST types); modle 3 (propensity score). All hypotheses were tested using a two-sided test with $\alpha=$ $0.05 . P \leq 0.05$ was considered statistically significant.

\section{RESULTS}

Between 10th October 2016 and 25th June 2019, 4,592 consecutive patients with minor acute ischemic stroke or highrisk TIA within $48 \mathrm{~h}$ of onset were included in the BRIDGE registry. A total of 152 patients were excluded because of incomplete information, of which 12 were missing important baseline information (age, sex, height, and weight), and 140 were missing important follow-up information (mRS scores at discharge and day 90). A total of 2,966 cases were entered into the analytical dataset based on the definition of the analytical dataset (Supplementary Figure 1), of which there were 1,042 cases in the NBP-compliance group and 1,924 cases in the NBP-noncompliance group. Table 1 shows the baseline characteristics in the two groups.

Of the 2,966 patients included in this study, 2,751 (92.7\%) were treated with antiplatelet therapy, of which 1,229 (43.80\%) were treated with aspirin only, 389 (13.11\%) with clopidogrel only, 1,233 (41.57\%) with dual antiplatelet, and 215 (7.25\%) were not treated with any antiplatelet agents.

The mean age of the NBP-non-compliance and the NBP compliance group was $65.00(56.00-73.00)$ and 64.00 (55.00$72.00)$ years. The median onset time from onset to hospital admission was $10.12(3.00-24.00) \mathrm{h}$ and $10.01(2.52-24.00) \mathrm{h}$ for the NBP-non-compliance group and the NBP compliance group, respectively. The median NIHSS score at admission was 2 for each of the two groups. The remaining baseline characteristics are detailed in Table 1.

\section{Primary Outcomes}

The proportion of patients with a favorable 90-day functional outcome (mRS 0-1) was higher in the NBP-compliance group $(92.05 \%, 949$ cases) than in the NBP-non-compliance group (87.42\%, 1,626 cases) $(\mathrm{OR}=1.67,95 \% \mathrm{CI}, 1.28-2.17$, and $p=$ 0.0002). In logistic regression model 1 , after adjusting for age and sex, there was a significant difference in the proportion 
TABLE 1 | Baseline characteristics between NBP compliance and NBP-non-compliance group.

\begin{tabular}{|c|c|c|c|c|}
\hline Variate & ALL & NBP-non-compliance group $(n=1,924)$ & NBP compliance group ( $n=1,042)$ & $\boldsymbol{P}$ \\
\hline Age, years & & $65.00(56.00-73.00)$ & $64.00(55.00-72.00)$ & 0.0779 \\
\hline Male & 1,935 (65.23\%) & $1,234(64.14)$ & $701(67.27)$ & 0.0868 \\
\hline Onset to admission time (h) & & $10.12(3.00-24.00)$ & $10.01(2.52-24.00)$ & 0.0283 \\
\hline Diabetes, $N(\%)$ & $763(25.72)$ & $486(25.26)$ & $277(26.58)$ & 0.4311 \\
\hline Hypertension, N (\%) & $1,954(65.88)$ & $1,270(66.01)$ & $684(65.64)$ & 0.8412 \\
\hline Dyslipidemia, N (\%) & $2,707(91.27)$ & $1,762(91.58)$ & 945 (90.69) & 0.4129 \\
\hline Stroke history, N (\%) & $110(3.7)$ & $70(3.64)$ & $40(3.84)$ & 0.7827 \\
\hline Smoke, $N(\%)$ & $735(24.78)$ & $469(24.38)$ & $266(25.53)$ & 0.0175 \\
\hline Atrial fibrillation, $N(\%)$ & $80(2.70)$ & $66(3.43)$ & $20(1.92)$ & 0.0192 \\
\hline Coronary heart disease, $N(\%)$ & $374(12.61)$ & $229(11.90)$ & $145(13.92)$ & 0.1148 \\
\hline Previous anti-hypertension, $N(\%)$ & $1,415(47.71)$ & 941 (48.91) & $474(45.49)$ & 0.0751 \\
\hline Previous antiplatelet, $N$ (\%) & $593(20.00)$ & $371(19.28)$ & $222(21.31)$ & 0.1886 \\
\hline NIHSS, median (IQR) & $2.00(1.00-4.00)$ & $2.00(1.00-3.00)$ & $2.00(1.00-4.00)$ & 0.0003 \\
\hline \multicolumn{5}{|l|}{ Current medication therapy } \\
\hline Antiplatelet, N (\%) & $2,751(92.75)$ & $714(92.13)$ & 2,037 (92.97) & 0.4371 \\
\hline Anticoagulant, N (\%) & $240(8.10)$ & $61(7.87)$ & $179(8.17)$ & 0.7932 \\
\hline Stain therapy, N (\%) & $2,447(82.50)$ & 625 (80.65) & $1,822(83.16)$ & 0.1135 \\
\hline Anti-hypertension, N (\%) & $1,297(43.73)$ & 359 (46.32) & $938(42.81)$ & 0.0903 \\
\hline
\end{tabular}

Values expressed as no./total no. (\%) unless otherwise indicated. mRS, modified rankin scale; NIHSS, National Institutes of Health Stroke Scale.

of favorable functional outcomes between the two groups (OR $=1.63,95 \% \mathrm{CI}, 1.25-2.13$, and $p=0.0003$ ). In the logistic regression model 2, after adjusting for age, sex, history of hypertension, body mass index, diabetes, dyslipidemia, smoking, alcohol consumption, TOAST types, and admission NIHSS score, the proportion of favorable functional outcomes remained significantly different between the two groups $(\mathrm{OR}=2.00$, 95\% CI, 1.50-2.65, and $p<0.0001$; Table 2). After adjusting for propensity score in model 3 , we found the similar results between the two groups [OR $=1.97(1.5-2.59), p<0.0001]$. The distribution of the modified Rankin scale scores between NBP-compliance and NBP-non-compliance group was shown in Figure 2.

\section{Key Secondary and Other Efficacy Outcomes}

The recurrence rate of ischemic stroke within 90 days was significantly higher in the NBP-compliance group than the NBPnon-compliance group (2.4 vs. $0.31 \%, \mathrm{OR}=7.78,95 \% \mathrm{CI}$, 3.19-18.95, and $P<0.0001$; Table 2). The incidence of death within 3 months was 0 cases $(0.00 \%)$ in the NBP-compliance group and 4 cases $(0.21 \%)$ in the NBP-non-compliance group (Table 2). Among all TIA patients, only one patient had stroke recurrence, and this patient was in the NBP-compliance group $(p=0.30)$.

\section{Bleeding Events}

The primary safety endpoint major bleeding occurred rarely and was not different between the groups $(0.29 \%$ NBP-compliance group and $0.0 \%$ NBP-non-compliance group).

\section{Subgroup Analysis}

The high proportion of 90-day favorable functional outcomes in the NBP-compliance group was consistent across the major subgroups, with interaction $p>0.05$, except that the 90 -day favorable functional outcome was better in the NIHSS (35) subgroup $(88.82 \%, 453$ cases) than in the NIHSS (0-2) subgroup (95.20\%, 496 cases) $(\mathrm{OR}=2.52,95 \% \mathrm{CI}, 1.81-3.50$, and interaction $p=0.00$ ), after adjustment for age, sex, history of hypertension, body mass index, diabetes, dyslipidemia, smoking, alcohol consumption, and admission NIHSS score (Table 3).

\section{DISCUSSION}

The principal result of this study is that compliance to NBPtreatment increased 90 days favorable functional outcome rate by $4.63 \%$ compared with the NBP-non-compliance patients (OR $=1.86,95 \% \mathrm{CI}, 1.41-2.45$ ) in non-disabling ischemic stroke or TIA patients, suggesting that the regular use of NBP in patients with minor stroke and high-risk TIA may improve functional outcomes and reduce disability. A post-hoc subgroup analysis raises the hypothesis that patients with NIHSS scores 3-5 may achieve a higher proportion of favorable functional outcomes at 90 days among patients with NIHSS scores $0-5$. This result suggests that NBP treatment may reduce the rate of disability in patients with potentially disabling minor strokes.

\section{NBP Compliance and Functional Outcome}

Our study suggested a recent substantial-high proportion of favorable functional outcomes $(86.8 \%, 2,577 / 2,966)$ in patients with non-disabling minor ischemic stroke or TIA. However, cohort studies suggested about $30 \%$ of patients with minor stroke had a functional disability at 90 days after stroke despite 
TABLE 2 | Outcome in the bridge trial according to compliance of nbp treatment.

\begin{tabular}{|c|c|c|c|c|c|c|c|c|}
\hline & $\begin{array}{c}\text { NBP-non } \\
\text {-compliance } \\
\text { group }\end{array}$ & $\begin{array}{c}\text { NBP } \\
\text { compliance } \\
\text { group }\end{array}$ & No adjusted & $P$ & Model 1 & $\boldsymbol{P}$ & Model 2 & $P$ \\
\hline & $n(\%)$ & $n(\%)$ & HR/OR (95\% Cl) & & HR/OR (95\% Cl) & & HR/OR (95\% Cl) & \\
\hline mRS (0-1) 90-day & 1,626 (87.42) & 949 (92.05) & $1.67(1.28-2.17)$ & 0.0002 & 1.63 (1.25-2.13) & 0.0003 & $1.86(1.41-2.45)$ & $<0.0001$ \\
\hline Stroke recurrence & $6(0.31)$ & $25(2.40)$ & 7.78 (3.19-18.95) & $<0.0001$ & 7.65 (3.14-18.66) & $<0.0001$ & 7.85 (3.22-19.18) & $<0.0001$ \\
\hline $\begin{array}{l}\text { Intracranial } \\
\text { hemmorrhage }\end{array}$ & $0(0.00)$ & $3(0.29)$ & - & - & - & - & - & - \\
\hline Death & $4(0.21)$ & $0(0.00)$ & - & - & - & - & - & - \\
\hline
\end{tabular}

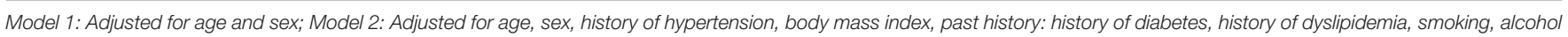
consumption, and admission NIHSS score.

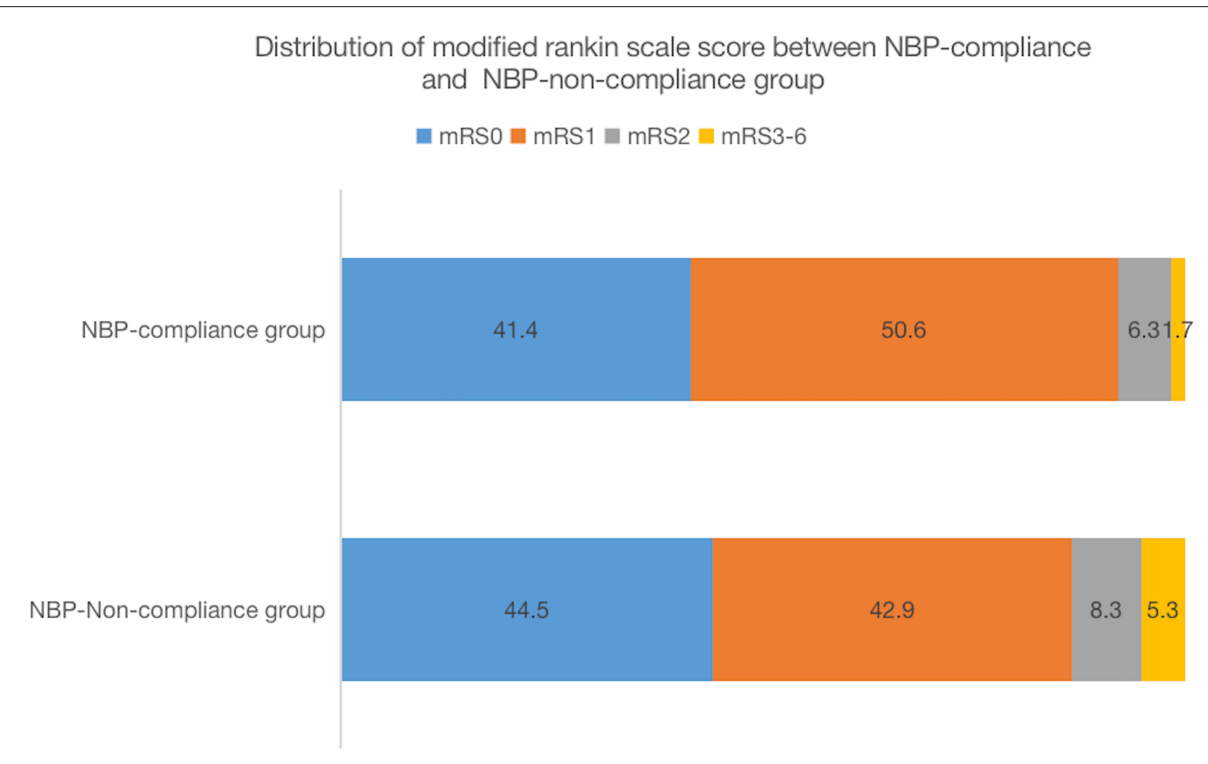

FIGURE 2 | Distribution of modified rankin scale score between NBP-compliance and NBP-non-compliance group.

nonsevere deficits at presentation $(16,17)$. The most likely reason for the discrepancy between previous cohort studies and the BRIDGE trial is the different inclusion criteria. Patients requiring intravenous thrombolysis and emergency revascularization were excluded from the present study; thus, many patients with potentially disabling stroke and large vessel stenosis or occlusion were excluded. Data from the United States showed that more than one-half of acute ischemic stroke hospitalizations had minor deficits, accounting for 4 of every 10 intravenous thrombolysis therapy (IVT) and 1 of every 10 mechanical thrombectomy treatments (18). A nationwide study in the United States, the excellent functional outcome only occurred in $48.2 \%$ of all minor strokes who underwent IVT or endovascular therapy (EVT) (18). The exclusion of EVT and IVT minor ischemic stroke or TIA patients from the present study might contribute to the substantial-high proportion of favorable functional outcomes. Only non-disabling minor stroke patients, rather than all minor stroke patients, were enrolled in this study was another contributor to the substantial-high favorable functional outcome rate. Our study suggested treatment with NBP, in addition to other standard treatments, may result in an additional good functional outcome in non-disabling ischemic stroke patients with no indication for EVT or IVT. This finding may be relevant to the pharmacological mechanism of NBP.

\section{Mechanism of NBP in Stroke}

$\mathrm{NBP}$ is a synthetic compound that has been approved for the treatment of ischemic stroke in China. The underlying mechanisms of the NBP-treatment efficacy have been reported in multiple studies. Animal experiments have shown that NBP can significantly improve the collateral circulation: rapidly opening the secondary collateral circulation, restoring the diameter of meningeal micro-arteries in the ischemic area and increasing blood flow velocity (19); establishing the tertiary collateral circulation, promoting the expression of VEGF and promoting vascular neovascularization (20). Recent animal studies have shown that daily intranasal NBP treatment can stimulate neurogenesis and angiogenesis in mice with ischemic 
TABLE 3 | Subgroup analysis on favorable functional outcome at 90 days (mRS 0-1).

\begin{tabular}{|c|c|c|c|c|}
\hline & NBP-non-compliance group, $n(\%)$ & NBP compliance group, $n$ (\%) & ${ }^{*}$ Adjusted OR (95\% Cl) & $P$ for interaction \\
\hline \multicolumn{5}{|l|}{ Sex } \\
\hline Male & $680(88.43)$ & $418(92.68)$ & $2.06(1.32-3.19)$ & 0.4671 \\
\hline Female & $372(86.11)$ & $183(90.59)$ & $1.59(0.89-2.84)$ & \\
\hline \multicolumn{5}{|l|}{ Age } \\
\hline$<60$ & $343(90.03)$ & $215(95.98)$ & $3.01(1.36-6.64)$ & 0.1580 \\
\hline$<25 \mathrm{~kg} / \mathrm{m}^{2}$ & $1,052(87.59)$ & $601(92.04)$ & $1.90(1.34-2.70)$ & 0.8304 \\
\hline$\geq 25 \mathrm{~kg} / \mathrm{m}^{2}$ & $574(87.10)$ & $348(92.06)$ & $1.79(1.13-2.84)$ & \\
\hline \multicolumn{5}{|c|}{ Hypertension } \\
\hline No & $384(87.67)$ & $221(94.04)$ & $2.36(1.25-4.47)$ & 0.4183 \\
\hline Yes & $668(87.55)$ & $380(90.91)$ & $1.75(1.15-2.67)$ & \\
\hline \multicolumn{5}{|c|}{ Dyslipidemia } \\
\hline No & $69(82.14)$ & $52(94.55)$ & $3.92(0.97-15.80)$ & 0.2053 \\
\hline Yes & $983(88.00)$ & $549(91.81)$ & $1.77(1.23-2.54)$ & \\
\hline \multicolumn{5}{|l|}{ Smoking } \\
\hline Yes & $799(87.23)$ & $445(90.63)$ & $1.60(1.09-2.34)$ & 0.0586 \\
\hline No & $253(88.77)$ & $156(96.30)$ & $3.96(1.55-10.09)$ & \\
\hline \multicolumn{5}{|c|}{ Alcohol consumption } \\
\hline No & $807(87.24)$ & $438(90.50)$ & $1.61(1.10-2.36)$ & 0.0590 \\
\hline Yes & $245(88.77)$ & $163(96.45)$ & $4.26(1.67-10.85)$ & \\
\hline \multicolumn{5}{|c|}{ Admission NIHSS score } \\
\hline 0 & $296(97.69)$ & $115(96.64)$ & $0.53(0.15-1.92)$ & 0.1151 \\
\hline
\end{tabular}

*Adjusted for age, sex, body mass index, past history: history of hypertension, history of diabetes, history of dyslipidemia, smoking, alcohol consumption, and admission NIHSS score.

strokes (21). NBP might address different pathophysiological functions, including anti-oxidation, anti-inflammatory, antiapoptosis, antithrombotic, and mitochondrial protection for acute ischemic stroke treatment (22).

Furthermore, another randomized clinical trial included 170 patients and found that NBP significantly increased circulating levels of endothelial progenitor cells in acute ischemic stroke patients and improved clinical outcomes (mRS at 90 days) (12).

\section{NBP Compliance and Stroke Recurrence}

Data from the CNSR study (the China National Stroke Registry) in China (from 2007 to 2008) showed that the 3-month recurrence rate of cerebrovascular disease (included ischemic stroke, intracranial hemorrhage, and subarachnoid hemorrhage) among patients with minor stroke and TIA was 9.8\% (23). However, the proportion of patients with minor stroke or TIA who had a 90 -day recurrence was only $1.04 \%(31 / 2,966)$ in this study cohort, lower than in previous studies (23). This discrepancy could be attributed to several possible reasons. First of all, previous reports of stroke risk after TIA is highest in the first 7 days and in patients without immediate treatment (11.0\%; 95\% CI, 8.6-13.5\%) (24). Over the past two decades, new treatment strategies and early management of TIA and minor strokes have significantly reduced stroke recurrence rates (25). The lowest risks were seen in studies of emergency treatment in specialist stroke services [0.9\% (95\% CI, 0.0-1.9)] (26-29) and the highest risks in population-based studies without urgent treatment [11.0\% (8.6-13.5)] (30-32). There was substantial heterogeneity between studies. Furthermore, in a most recent 
systematic review and meta-analysis of 206,455 patients in 68 unique studies during 5 decades, the subsequent risk of ischemic stroke was $4.7 \%$ within 90 days and the incidence was gradually decreased by the time of trials, among the study population recruited before 1999 was significantly higher (25). Second of all, the definition of stroke recurrence varies between studies, resulting in different incidences of observed stroke recurrence. One study showed a 90-day recurrence rate of 20/180 for patients with minor stroke and TIA, but a real new stroke (infarction at a new site) of only $2 / 180$ was found at 30 -day follow-up using MRI (33). Thirdly, the dual antiplatelet therapy in the present study $(41.57 \%)$ and the combined treatment with NBP may also have contributed to the lower stroke recurrence rate. The present study found that the 90 days ischemic stroke recurrence rate was significantly higher in the NBP-compliance group than in the NBP-non-compliance group. However, due to the low overall event rate and the short follow-up period, we cannot rule out the possibility of an incidental finding. A large-scale study with a more extended follow-up period is needed to explore this finding.

\section{Medication Adherence}

In the present study, $80 \%$ was used as the cutoff value of NBP drug compliance. Previous observational studies that evaluated the relationship between medication compliance and outcome used $80-100 \%$ as an indicator of high medication compliance (15), and found that it was associated with better blood pressure control (34) and blood sugar control (35). Furthermore, pharmacy supplement data and patient self-reports are the most commonly used methods of compliance assessment which is similar to the present study (15).

Despite a higher rate of recurrences, patients in the compliance group had a greater proportion of functional outcomes than patients in the non-compliance group. This discrepancy is due to the fact that patients in the compliance group with recurrent strokes still have a good functional outcome after 90 days. In this study, both the NBP-compliance and noncompliance groups had recurrent stroke cases of 25 and 6, respectively. However, the proportion of 90-day good functional outcomes in the NBP-compliance group was $64.00 \%(16 / 25)$, compared with $33.33 \%(2 / 6)$ in the NBP-non-compliance group (Supplementary Table 1). We also noticed that in the NBPcompliance group, $36.00 \%$ (9/25) of stroke recurrence occurred in patients with baseline NIHSS0-1 and TIA in the NBPnon-compliance group, and 50.00\% (3/6) in NBP compliance group. The interaction between baseline NIHSS score and 90day functional outcomes might be related to the relatively high proportion of good functional outcomes in the NBP compliance group.

The strengths of this study are mainly that, (1) the data collected prospectively in 51 cities in China represent the Chinese population with non-disabling minor stroke and TIA. (2) This study was the largest data analysis based on the efficacy analysis of treatment with NBP in the Chinese nondisabling minor stroke and TIA population. (3) In patients who are not suitable for intravenous thrombolysis or acute endovascular thrombectomy therapy, NBP treatment might improve functional outcomes at 90 days, providing a potential alternative treatment for non-disabling minor stroke and TIA patients.

The limitations of this study are, (1) that the patients enrolled were not classified for stroke etiology, such as the proportion of patients with symptomatic large vessel occlusion, and minor ischemic strokes with large vessel occlusions may be associated with higher stroke recurrence and progression. (2) The present study used 90-day mRS as the primary outcome, and there is a possibility that a longer follow-up period may have identified more stroke recurrence. (3) The treatment strategy for both groups were determined by the physician based on the patient's condition. Potentially disabling patients or patients with large vessel occlusion have been excluded due to administration of thrombolytic therapy or endovascular thrombectomy, so the present study did not reflect the characteristics of the overall minor stroke and TIA patients cohort. Another shortcoming of this study is that patients who were not compliant with NBP may also be non-compliant with other medications. Adherence to antithrombotic, lipid-lowering, and antihypertensive drugs was ensured during follow-up in our study, but adherence to glucose-lowering drugs was not followed up in this study.

\section{CONCLUSION}

In this real-world experience, patients who complied with NBP therapy gained better short-term functional outcomes than those who did not, and the efficacy might even be more in those patients with NIHSS score from 3 to 5 . Further large scale randomized controlled trial to explore the association between NBP therapy and non-disabling minor acute ischemic stroke or TIA outcomes is warranted in the coming future.

\section{DATA AVAILABILITY STATEMENT}

The original contributions presented in the study are included in the article/Supplementary Material, further inquiries can be directed to the corresponding author/s.

\section{ETHICS STATEMENT}

The studies involving human participants were reviewed and approved by the Medical Ethics Council of the First Affiliated Hospital of Jinan University. The patients/participants provided their written informed consent to participate in this study.

\section{AUTHOR CONTRIBUTIONS}

ZT and AX conceived and designed the study. YZ, SH, and YD performed the study. YZ and $\mathrm{ZT}$ analyzed the 
data. ZT wrote the paper. AX gave suggestions how to design the study, edit the results, and write the manuscript. All authors contributed to the article and approved the submitted version.

\section{ACKNOWLEDGMENTS}

The authors would like to thank Science and Technology Plan Project of Tianhe District, Guangzhou City, Guangdong Province, China. No. 2018YT006 and Cultivating fund of the

\section{REFERENCES}

1. Johnston SC, Gress DR, Browner WS, Sidney S. Short-term prognosis after emergency department diagnosis of TIA. JAMA. (2000) 284:29016. doi: 10.1001/jama.284.22.2901

2. Coull AJ, Lovett JK, Rothwell PM, Oxford Vascular Study. Population based study of early risk of stroke after transient ischaemic attack or minor stroke: implications for public education and organisation of services. BMJ. (2004) 328:326. doi: 10.1136/bmj.37991.635266.44

3. Hill MD, Yiannakoulias N, Jeerakathil T, Tu JV, Svenson LW, Schopflocher DP. The high risk of stroke immediately after transient ischemic attack: a population-based study. Neurology. (2004) 62:2015-20. doi: 10.1212/01.WNL.0000129482.70315.2F

4. Eliasziw M, Kennedy J, Hill MD, Buchan AM, Barnett HJ, North American Symptomatic Carotid Endarterectomy Trial Group. Early risk of stroke after a transient ischemic attack in patients with internal carotid artery disease. CMAJ. (2004) 170:1105-9. doi: 10.1503/cmaj.1030460

5. Wang Y, Wang Y, Zhao X, Liu L, Wang D, Wang C, et al. Clopidogrel with aspirin in acute minor stroke or transient ischemic attack. $N$ Engl J Med. (2013) 369:11-9. doi: 10.1056/NEJMoa1215340

6. Ma H, Campbell B, Parsons MW, Churilov L, Levi CR, Hsu C, et al. Thrombolysis guided by perfusion imaging up to 9 hours after onset of stroke. N Engl J Med. (2019) 380:1795-803. doi: 10.1056/NEJMoa1813046

7. Goyal M, Demchuk AM, Menon BK, Eesa M, Rempel JL, Thornton J, et al. Randomized assessment of rapid endovascular treatment of ischemic stroke. N Engl J Med. (2015) 372:1019-30. doi: 10.1056/NEJMoa1414905

8. Albers GW, Marks MP, Kemp S, Christensen S, Tsai JP, Ortega-Gutierrez S, et al. Thrombectomy for stroke at 6 to 16 hours with selection by perfusion imaging. N Engl J Med. (2018) 378:708-18. doi: 10.1056/NEJMoa1713973

9. Cui LY, Zhu YC, Gao S, Wang JM, Peng B, Ni J, et al. Ninetyday administration of dl-3-n-butylphthalide for acute ischemic stroke: a randomized, double-blind trial. Chin Med J (Engl). (2013) 126:3405-10. doi: 10.3760/cma.j.issn.0366-6999.20123240

10. Lu XL, Luo D, Yao XL, Wang GL, Liu ZY, Li ZX, et al. dl-3nButylphthalide promotes angiogenesis via the extracellular signal-regulated kinase $1 / 2$ and phosphatidylinositol 3-kinase/Akt-endothelial nitric oxide synthase signaling pathways. J Cardiovasc Pharmacol. (2012) 59:35262. doi: 10.1097/FJC.0b013e3182443e74

11. Huang XX, Hu D, Qu ZW, Zhang JT, Feng YP. [Effect of dl-3-butylphthalide on the striatum extracellular amino acid and dopamine contents in the rat during cerebral ischemia]. Yao Xue Xue Bao. (1996) 31:246-9.

12. Zhao H, Yun W, Zhang Q, Cai X, Li X, Hui G, et al. Mobilization of circulating endothelial progenitor cells by dl-3-n-butylphthalide in acute ischemic stroke patients. J Stroke Cerebrovasc Dis. (2016) 25:75260. doi: 10.1016/j.jstrokecerebrovasdis.2015.11.018

13. Liu L, Ding J, Leng X, Pu Y, Huang LA, Xu A, et al. Guidelines for evaluation and management of cerebral collateral circulation in ischaemic stroke 2017. Stroke Vasc Neurol. (2018) 3:117-30. doi: 10.1136/svn-2017-000135

14. Mäenpää H, Manninen V, Heinonen OP. Comparison of the digoxin marker with capsule counting and compliance questionnaire methods for measuring affiliated Shun De hospital of Jinan University for their funding of this research.

\section{SUPPLEMENTARY MATERIAL}

The Supplementary Material for this article can be found online at: https://www.frontiersin.org/articles/10.3389/fneur. 2021.720664/full\#supplementary-material

Supplementary Figure 1 | Trial flow diagram.

Supplementary Table 1 | Proportion of 90 day good functional outcomes in patients with stroke recurrence.

compliance to medication in a clinical trial. Eur Heart J. (1987) 8(Suppl 1):39-43. doi: 10.1093/eurheartj/8.suppl_I.39

15. Ho PM, Bryson CL, Rumsfeld JS. Medication adherence: its importance in cardiovascular outcomes. Circulation. (2009) 119:3028-35. doi: 10.1161/CIRCULATIONAHA.108.768986

16. Fischer U, Baumgartner A, Arnold M, Nedeltchev K, Gralla J, De Marchis GM, et al. What is a minor stroke. Stroke. (2010) 41:6616. doi: 10.1161/STROKEAHA.109.572883

17. Khatri P, Conaway MR, Johnston KC. Ninety-day outcome rates of a prospective cohort of consecutive patients with mild ischemic stroke. Stroke. (2012) 43:560-2. doi: 10.1161/STROKEAHA.110.593897

18. Saber H, Khatibi K, Szeder V, Tateshima S, Colby GP, Nour $\mathrm{M}$, et al. Reperfusion therapy frequency and outcomes in mild ischemic stroke in the United States. Stroke. (2020) 51:32419. doi: 10.1161/STROKEAHA.120.030898

19. Qin C, Zhou P, Wang L, Mamtilahun M, Li W, Zhang Z, et al. Dl-3$\mathrm{N}$-butylphthalide attenuates ischemic reperfusion injury by improving the function of cerebral artery and circulation. J Cereb Blood Flow Metab. (2019) 39:2011-21. doi: 10.1177/0271678X18776833

20. Yang CS, Guo A, Li Y, Shi K, Shi FD, Li M. Dl-3-n-butylphthalide reduces neurovascular inflammation and ischemic brain injury in mice. Aging Dis. (2019) 10:964-76. doi: 10.14336/AD.2019.0608

21. Qu M, Zhao J, Zhao Y, Sun J, Liu L, Wei L, et al. Vascular protection and regenerative effects of intranasal DL-3-N-butylphthalide treatment after ischaemic stroke in mice. Stroke Vasc Neurol. (2020). doi: 10.1136/svn-2020-000364

22. Wang S, Ma F, Huang L, Zhang Y, Peng Y, Xing C, et al. Dl-3-n-butylphthalide (NBP): a promising therapeutic agent for ischemic stroke. CNS Neurol Disord Drug Targets. (2018) 17:338-47. doi: 10.2174/1871527317666180612125843

23. Wu L, Wang A, Wang X, Zhao X, Wang C, Liu L, et al. Factors for short-term outcomes in patients with a minor stroke: results from China National Stroke Registry. BMC Neurol. (2015) 15:253. doi: 10.1186/s12883-015-0505-z

24. Giles MF, Rothwell PM. Risk of stroke early after transient ischaemic attack: a systematic review and meta-analysis. Lancet Neurol. (2007) 6:106372. doi: 10.1016/S1474-4422(07)70274-0

25. Shahjouei S, Sadighi A, Chaudhary D, Li J, Abedi V, Holland N, et al. A 5-decade analysis of incidence trends of ischemic stroke after transient ischemic attack: a systematic review and meta-analysis. JAMA Neurol. (2020). doi: 10.1001/jamaneurol.2020.3627

26. Luengo-Fernandez R, Gray AM, Rothwell PM. Effect of urgent treatment for transient ischaemic attack and minor stroke on disability and hospital costs (EXPRESS study): a prospective population-based sequential comparison. Lancet Neurol. (2009) 8:235-43. doi: 10.1016/S1474-4422(09)70019-5

27. Cucchiara BL, Messe SR, Taylor RA, Pacelli J, Maus D, Shah $\mathrm{Q}$, et al. Is the $\mathrm{ABCD}$ score useful for risk stratification of patients with acute transient ischemic attack. Stroke (2006) 37:1710-4. doi: 10.1161/01.STR.0000227195.46336.93

28. Calvet D, Lamy C, Touzé E, Oppenheim C, Meder JF, Mas JL. Management and outcome of patients with transient ischemic attack admitted to a stroke unit. Cerebrovasc Dis. (2007) 24:80-5. doi: 10.1159/000103120 
29. Lavallée PC, Meseguer E, Abboud H, Cabrejo L, Olivot JM, Simon $\mathrm{O}$, et al. A transient ischaemic attack clinic with round-the-clock access (SOS-TIA): feasibility and effects. Lancet Neurol. (2007) 6:95360. doi: 10.1016/S1474-4422(07)70248-X

30. Rothwell PM, Giles MF, Flossmann E, Lovelock CE, Redgrave JN, Warlow $\mathrm{CP}$, et al. A simple score (ABCD) to identify individuals at high early risk of stroke after transient ischaemic attack. Lancet. (2005) 366:2936. doi: 10.1016/S0140-6736(05)66702-5

31. Correia M, Silva MR, Magalhães $R$, Guimarães L, Silva MC. Transient ischemic attacks in rural and urban northern Portugal: incidence and short-term prognosis. Stroke. (2006) 37:50-5. doi: 10.1161/01.STR.0000195209.26543.8f

32. Lovett JK, Dennis MS, Sandercock PA, Bamford J, Warlow CP, Rothwell PM. Very early risk of stroke after a first transient ischemic attack. Stroke. (2003) 34:e138-40. doi: 10.1161/01.STR.0000080935.01264.91

33. Coutts SB, Hill MD, Campos CR, Choi YB, Subramaniam S, Kosior JC, et al. Recurrent events in transient ischemic attack and minor stroke: what events are happening and to which patients. Stroke. (2008) 39:24616. doi: 10.1161/STROKEAHA.107.513234

34. Bramley TJ, Gerbino PP, Nightengale BS, Frech-Tamas F. Relationship of blood pressure control to adherence with antihypertensive monotherapy in 13 managed care organizations. J Manag Care Pharm. (2006) 12:23945. doi: 10.18553/jmcp.2006.12.3.239
35. Ho PM, Rumsfeld JS, Masoudi FA, McClure DL, Plomondon ME, Steiner JF, et al. Effect of medication nonadherence on hospitalization and mortality among patients with diabetes mellitus. Arch Intern Med. (2006) 166:183641. doi: $10.1001 /$ archinte.166.17.1836

Conflict of Interest: The authors declare that the research was conducted in the absence of any commercial or financial relationships that could be construed as a potential conflict of interest.

Publisher's Note: All claims expressed in this article are solely those of the authors and do not necessarily represent those of their affiliated organizations, or those of the publisher, the editors and the reviewers. Any product that may be evaluated in this article, or claim that may be made by its manufacturer, is not guaranteed or endorsed by the publisher.

Copyright (c) 2021 Tan, Zhao, Yang, He, Ding and Xu. This is an open-access article distributed under the terms of the Creative Commons Attribution License (CC BY). The use, distribution or reproduction in other forums is permitted, provided the original author(s) and the copyright owner(s) are credited and that the original publication in this journal is cited, in accordance with accepted academic practice. No use, distribution or reproduction is permitted which does not comply with these terms. 